\title{
RBD and ACE2 Embedded Chitosan Nanoparticles as a Prevention Approach for SARS-COV 2
}

\author{
Farmanli Orkhan ${ }^{1}$, Uysal Melike ${ }^{2 *}$, Gokdemir Cihan ${ }^{3}$, Donmez Omer Faruk ${ }^{4}$, Bastug Samet ${ }^{5}$, \\ Uckun Ilknur ${ }^{6}$ and Jafarov Alemdar ${ }^{7}$ \\ ${ }^{1}$ Department of Pathology, Marmara University School of Medicine,Turkey
}

${ }^{2}$ Department of Biotechnology, Institute of Health Sciences, Bezmialem Vakif University, Turkey

${ }^{3}$ Faculty of Medicine, Marmara University, Turkey

${ }^{4}$ Faculty of Medicine, Istanbul Medeniyet University, Turkey

${ }^{5}$ Faculty of Pharmacy, Istanbul University, Turkey

${ }^{6}$ Faculty of Medicine, Kırlkkale University, Turkey

${ }^{7}$ Department of Hematology, Azerbaijan Medical University, Turkey

*Corresponding author: Uysal Melike, Department of Biotechnology, Institute of Health Sciences, Bezmialem Vakif University, Turkey

\section{ARTICLE INFO}

Received: 戛 July 02, 2021

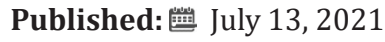

Citation: Farmanli Orkhan, Uysal Melike, Gokdemir Cihan, Donmez Omer Faruk, Bastug Samet, et al., RBD and ACE2 Embedded Chitosan Nanoparticles as a Prevention Approach for SARS-COV 2. Biomed J Sci \& Tech Res 37(2)-2021. BJSTR. MS.ID.005960.

\begin{abstract}
The SARS-CoV-2 virus appeared in China in December 2019 and has quickly become a pandemic that has influenced the world. Researches have not yet brought a treatment method to the disease and in many countries, people are called to stay at home. In our study, we hypothesized about what preventive treatment mechanisms could be used until the vaccine that could be developed against this virus was found. The purpose of suggesting these hypotheses is to provide pretreatment to prevent people who have to work from becoming infected with the virus. In our hypotheses, we selected our drug delivery systems suitable for daily use to be prepared by biotechnological methods.
\end{abstract}

Keywords: Novel Coronavirus; SARS-CoV-2; Chitosan Nanoparticles; RBD; ACE2; Hypothesis

\section{Introduction}

A new type of coronavirus-associated persistent pneumonia outbreak called SARS-CoV-2, which causes severe acute respiratory syndrome, was reported in Wuhan, China in Hubei Province in December 2019. In the following weeks, infections spread rapidly to China and other countries around the world [1]. Coronaviruses (CoVs) are known to cause enteric and respiratory diseases in animals and humans, which are positive stranded RNA viruses that are not segmented into large envelopes. Most human CoVs such as hCoV - 229E, OC43, NL63 and HKU1 cause mild respiratory diseases, but two previously unknown CoVs, severe acute respiratory syndrome CoV (SARS - CoV) and Middle East respiratory syndrome CoV (MERS - The worldwide spread of CoV) has drawn global attention to the deadly potential of human CoVs. Genomic analysis shows that SARS - CoV - 2 belongs to the same betacoronavirus family as MERS - CoV and SARS - CoV and shares a sequence that shows a high homology with SARS - CoV. A cellular receptor angiotensin-converting enzyme 2 (ACE2) is mainly mediated by the introduction of SARS - CoV into human host cells. It is expressed in the human respiratory epithelium, lung parenchyma, vascular endothelium, kidney cells and small intestine cells [2]. 


\section{ACE2 Function as a Double-Edged Sword}

ACE2 protein is a carboxypeptidase which has an important function in the conversion of Angiotensin-1 to Angiotensin-(1-9) and generally found in lungs. ACE2's normal funtion is so important since the ratio of Angiotensin-1/Angiotensin-2 has major effect on sustainability of lung function [3]. Li et al. showed that ACE2 protein also has a role as a major attachment side of Coronaviruses [4]. These two important functions make ACE2 a double edged sword since its function is so crucial for protecting the lungs from lung failure [5]. However, ACE2 involvement in the infection mechanism of SARS-CoV viruses,makes ACE2 an important target of theurapatic approaches. So it becomes more necessary to focus on structural form of ACE2 and its components [6].

\section{ACE2 Distribution along Different Tissues}

ACE2 protein which is the main attachment side of SARS$\mathrm{CoV}$ has found in many different tissues. The mapping of ACE2 expression in different tissues can potentially identify the possible routes of infection for SARS-CoV, and possible routes of spread and replication throughout the body. One of the most important of these locations is the lung alveolar epithelial cells which causes the main symptom and signs of COVID19 and it is the responsible from high number of deaths [7]. Numerous variety of studies showed that ACE2 mRNA is highly expressed in renal, cardiovascular, and gastrointestinal tissues [8-10]. Hamming and colleagues study is one of the most important studies which shows the distribution of ACE2 along different types of tissues. The most striking result in this study, addition the previous studies results, is the demonstration of ACE2 expression in the basal layer of the non-keratinizing squamous epithelium of the nasal and oral mucosa and the nasopharynx [7].

\section{SARS-CoV-2 Cell Entry Mechanism}

A distinctive feature of coronaviruses is that they recognize a variety of receptors, including both protein receptors and sugar receptors. Coronaviruses enter cells first by recognizing a host cell surface receptor for viral binding and then creating an endosome. Receptors play an important role not only in viral binding but also in membrane fusion process [11]. In order for a viral infection to begin, a receptor expressed by host cells needs to bind to the virus ligand. ACE2 the host receptor identified by researchers working with SARS-CoV, is now claimed to be a receptor for COVID-19 [12]. One of the most important of these locations is the lung alveolar epithelial cells which causes the main symptom and signs of COVID19 and it is the responsible from high number of deaths [7] . Importantly, the sequence of the COVID-19 spike(S) protein receptor binding domain is similar to that of SARS-CoV, which caused a pandemic in 2003. Mutagenic analyses revealed the domains of SARS and ACE2 receptor which provides efficient interaction between these two. One of these studies is conducted by
Xiao et al.and results showed that an independently folded region called Receptor Binding Domain(RBD) which is in between the aminoacids of $318-510$ on SARS-Spike(SARS-S) protein, binds to ACE2 receptor with higher affinity than the other remaining parts of SARS-S protein [13]. S protein is the trimeric protein found in metastable inhibition conformation for fusion of viral membrane and host cell membrane. Each of the $\mathrm{S} 1$ subunits contains a receptor binding domain (RBD) that mediates receptor recognition. When the RBD is attached to the host cell, the balance of the trimeric structure is disturbed, which causes the $\mathrm{S} 1$ subunit to shed and the S2 subunit to change its conformation to take the form of postfusion [14]. The SARS-CoV-2 S1 / S2 region is believed to be cleaved by the cathepsin L, similar to the SARS-CoV [15]. Cell studies carried out by the researchers were predicted that after the spike protein that binds to ACE- 2 and then, the virus is introduced into the host cell via cathepsinB /L and TMPRSS2 proteases [16]. Although the newly introduced SARS-CoV-2 entry mechanisms and endocytic pathway are not fully known, it is known to use the ACE2 receptor, which is the same as SARS-CoV for viral entry. The sensitivity of SARS-CoV-2 to the inhibitory effect of chloroquine (CQ) suggests that this new $\mathrm{CoV}$ will use the same endocytic pathway for entry into host cells. SARS-CoV, seen as a possible endocytic pathway, enters the cell through autophagy. Autophagy is controlled by a group of proteins encoded by autophagy-related genes (ATG) in several successive stages [17].

\section{SARS-CoV-2 Structure}

A coronavirus have four structural proteins, including spike $(\mathrm{S})$, envelope (E), membrane (M), and nucleocapsid $(\mathrm{N})$ protein (Picture 1).S protein displays the most important roles, including viral attachment, fusion and entry [18]. The S protein provides viral particules entry into host cells. Firstly, it engages to a host receptor through the receptor-binding domain (RBD) in the S1 subunit. Subsequently, it fuses the viral and host membranes through the S2 subunit [19]. Therefore, it is critical to define the RBD in SARSCoV-2 $S$ protein for the development of virus attachment inhibitors, neutralizing antibodies, and vaccines [20]. There are more than one study on this subject in the literature. In a study belongs to Lan, J et al, they expressed the SARS-CoV-2 RBD (residues Arg319-Phe541) and the N-terminal peptidase domain of ACE2 (residues Ser19Asp615) in Hi5 insect cells and established the final model contains residues Thr333 to Gly526 of the SARS-CoV-2 RBD and residues Ser19 to Asp615 of the ACE2 N-terminal peptidase domain [21]. Furthermore, Tai et al. identified the region of SARS-CoV-2 RBD at residues 331 to 524 of S protein. Then, they made recombinant RBD protein using pFUSE-hIgG1-Fc2 expression vector, expressed the protein in mammalian cell 293T. They have demonstrated SARSCoV-2 RBD bound to sACE2 in a dose dependent manner. Moreover, they have also established, binding between SARS-CoV-2 RBD and sACE2 with $50 \%$ effective dose (EC50) was stronger than that 
between SARS-CoV RBD and SACE2. As a result of study, Tai et al., have suggested that SARS-CoV-2 RBD protein could be developed as an effective therapeutic agent against SARS-CoV-2 infection [20].
Ultimately, based on these studies, in our hypothesis, we decided to use RBD residues to bind to the ACE2 receptors on the host cells (Figure 1).

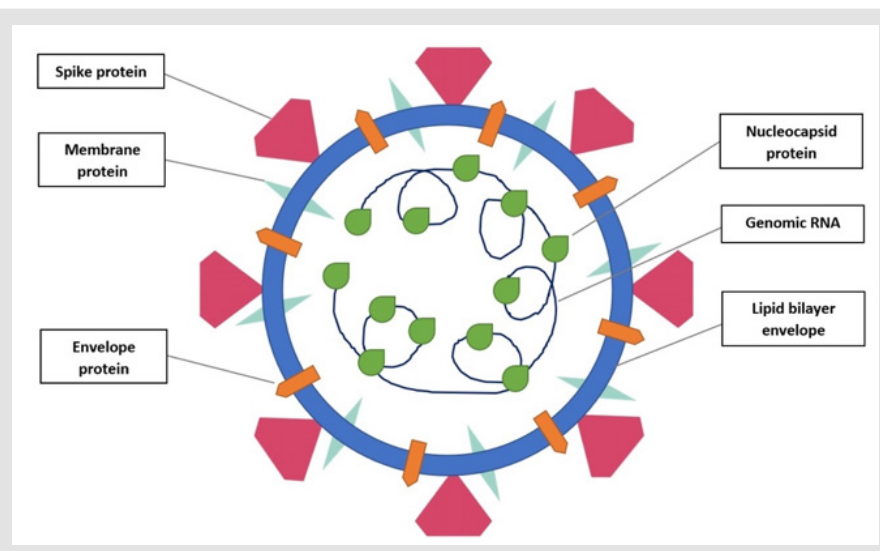

Figure 1: The Structure of SARS-CoV 2.

\section{Chitosan Nanoparticles}

Chitosan is a biocompatible, biodegradable polymer that is considered safe for use in the human diet and approved for dressing applications [22-24]. Chitosan polymer has been used as a transporter in polymeric nanoparticles for drug delivery by different routes of administration [25]. The Chitosan polymer has chemical functional groups that can be modified to achieve certain goals and make it a polymer with a wide range of potential applications in human health and various fields. The chitosan nanoparticles and chitosan derivatives nanoparticles have a positive surface charge and mucoadhesion properties that can stick to mucous membranes and release the drug load in continuous release [26]. Chitosanbased nanoparticles has several applications in non-parenteral drug delivery for cancer, lung diseases, gastrointestinal diseases, administration of the brain and treatment of ocular infections [27]. The Chitosan nanoparticles show low toxicity in both in vitro and some in vivo models.

\section{a. Modified Chitosan Nanoparticles with Antibody}

Drug delivery vehicles such as polymeric nanoparticles modified with specific ligands such as antibodies have been widely used for targeted therapy. When designing nanoparticle-antibody conjugates for drug delivery or medical applications, several properties of the structure of the nanoparticles are important. The NPs must be chemically and biologically inert, can be stable in physiological conditions, must move freely in the body, contain a surface that is easily conjugated to the targeting antibody [28]. Here, we design a new type of chitosan nanoparticles for treating the coronavirus disease, for this goal we'll binded the ACE2 receptor and RBD protein to the surface of chitosan nanoparticles by chemical method.

\section{The Fast and Effective Method: The Usage of Inhaler Spray}

Inhaled treatments give easy, stable and effective results in various lung diseases (eg: cystic fibrosis, asthma) [29]. Low doses and low side effects are among the advantages of this form. The most important advantage of inhalation over parenteral doses is that it does not require sterilization and is easy to apply. The drugs reach the bronchial muscles at a higher concentration than other systemic applications by inhalation and their effects are at maximum level. Since the local metabolism of inhaled drugs is slow, its effects last longer (Bizim makale). Another reason that we prefer treatment method through inhalation is the presence of ACE- 2 receptors in the nasopharyngeal region [7]. As a result of the literature review, we decided that inhaled therapies for lung and respiratory diseases are more effective at the local level and thus we planned our study accordingly.

\section{Hypothesis: RBD and ACE2 Embedded Chitosan Nanoparticles Prevent to Attach SARS CoV2 to Host Cells}

As plasma therapy and other medical treatments are insufficient and vaccination studies take a long time, we planned to develop an prevention therapy so that people can return to their daily life activities. In the literature searches, we established that ACE 2 was located in both the respiratory tract and nasopharyngeal epithelium. Considering the normal physiological function of Ace 2 , it is not a matter of discussion of complete inhibition of the receptors in this field. Therefore, we thought it could be enough to provide inhibition during the time people spend outside. In our study to provide an adjustable inhibition, we anticipated the use of chitosan nanoparticles that can be compatible with the body. 
We assumed that the virus RBD residues should be added to these nanoparticles. Thus, inhibition of host cells ACE2 will prevent the attachment of viruses. We also planned to add ACE2 antibody on nanoparticles. In this way, the nanoparticles will be attached to host cells with the RBD tip, and to viral particles with the ACE2 tip (Figure 2). Extensive studies on biocompatibility of chitosan nanoparticles in literature screening make it possible to test this experiment in a short time on humans. Moreover, it is easy to add antibodies to chitosan therefore we chose these nanoparticles. We anticipated the use of inhaler spray as a more specific and effective method for respiratory and nasopharyngeal receptors (Figure 2).

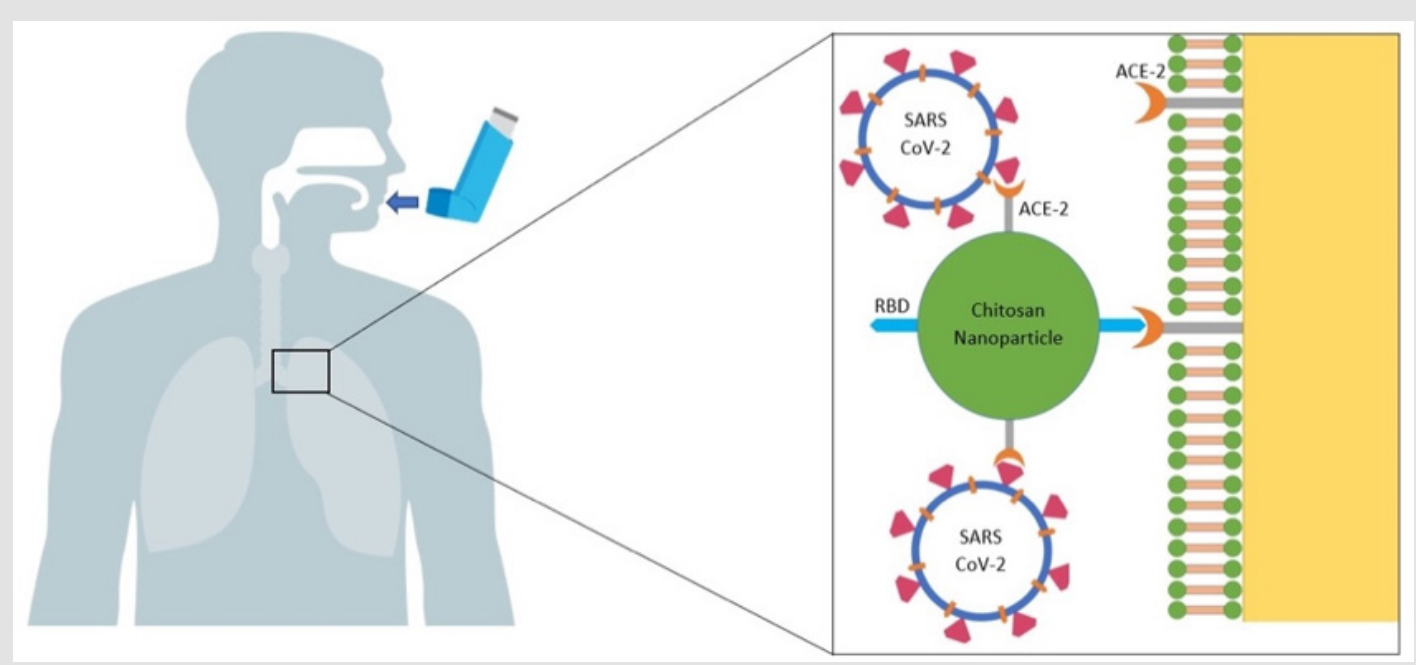

Figure 2: Attachment of modified chitosan nanoparticles to the host cell ACE 2 receptor and attachment of viruses to ACE 2 on the nanoparticle.

\section{Conflict of Interest}

None.

\section{References}

1. Zu ZY, Jiang MD, Xu PP, Chen W, Ni QQ, et al. (2020) Coronavirus Disease 2019 (COVID-19): A Perspective from China. Radiology 200490 (2020).

2. Li YC, Bai WZ, Hashikawa T (2020) The neuroinvasive potential of SARSCoV2 may play a role in the respiratory failure of COVID-19 patients. J Med Virol.

3. Recombinant human ACE2: acing out angiotensin II in ARDS therapy. Critical Care.

4. Li W, Moore MJ, Vasilieva N, Sui J, Wong SK, et al. (2003) Angiotensinconverting enzyme 2 is a functional receptor for the SARS coronavirus. Nature 426: 450 .

5. Kuba K, Imai Y, Ohto-Nakanishi T, Penninger JM (2010) Trilogy of ACE2: a peptidase in the renin-angiotensin system, a SARS receptor, and a partner for amino acid transporters. Pharmacol Ther 128: 119.

6. Zhang H, Penninger JM, Li Y, Zhong N, Slutsky AS (2020) Angiotensinconverting enzyme 2 (ACE2) as a SARS-CoV-2 receptor: molecular mechanisms and potential therapeutic target, Intensive Care Med.

7. Hamming I, Timens W, Bulthuis MLC, Lely AT, Navis GJ, et al. (2004) Tissue distribution of ACE2 protein, the functional receptor for SARS coronavirus. A first step in understanding SARS pathogenesis. J Pathol 203: 631 .

8. Harmer D, Gilbert M, Borman R, Clark KL (2002) Quantitative mRNA expression profiling of ACE 2, a novel homologue of angiotensin converting enzyme. FEBS Lett 532: 107.

9. Donoghue M, Hsieh F, Baronas E, Godbout K, Gosselin M, et al. (2000) A novel angiotensin-converting enzyme-related carboxypeptidase (ACE2) converts angiotensin I to angiotensin 1-9. Circ Res 87: E1.
10. Tipnis SR, Hooper NM, Hyde R, Karran E, Christie G, et al. (2000) A human homolog of angiotensin-converting enzyme. Cloning and functional expression as a captopril-insensitive carboxypeptidase. J Biol Chem 275: 33238

11. Shang J, Wan Y, Liu C, Yount B, Gully K, et al. (2020) Structure of mouse coronavirus spike protein complexed with receptor reveals mechanism for viral entry. PLoS Pathog 16.

12. Rothan HA, Byrareddy SN (2020) The epidemiology and pathogenesis of coronavirus disease (COVID-19) outbreak. J Autoimmun 109: 102433.

13. Xiao X, Chakraborti S, Dimitrov AS, Gramatikoff K, Dimitrov DS (2003) The SARS-CoV S glycoprotein: expression and functional characterization, Biochem. Biophys. Res. Commun 312: 1159.

14. Wrapp D, Wang N, Corbett KS, Goldsmith JA, Hsieh CL, et al. (2020) CryoEM structure of the 2019-nCoV spike in the prefusion conformation. Science 367: 1260.

15. Ashour HM, Elkhatib WF, Rahman MM, Elshabrawy HA (2020) Insights into the Recent 2019 Novel Coronavirus (SARS-CoV-2) in Light of Past Human Coronavirus Outbreaks. Pathogens 9: 186.

16. Hoffmann M, Kleine-Weber H, Schroeder S, Krüger N, Herrler T, et al. (2020) SARS-CoV-2 Cell Entry Depends on ACE2 and TMPRSS2 and Is Blocked by a Clinically Proven Protease Inhibitor. Cell.

17. Yang N, Shen HM (2020) Targeting the Endocytic Pathway and Autophagy Process as a Novel Therapeutic Strategy in COVID-19. Int J Biol Sci 16: 1724

18. Du L, He Y, Zhou Y, Liu S, Zheng BJ, et al. (2009) The spike protein of SARS-CoV--a target for vaccine and therapeutic development. Nat Rev Microbiol 7: 226.

19. Lu G, Hu Y, Wang Q, Qi J, Gao F, et al. (2013) Molecular basis of binding between novel human coronavirus MERS-CoV and its receptor CD26. Nature 500: 227. 
20. Characterization of the receptor-binding domain (RBD) of 2019 novel coronavirus: implication for development of RBD protein as a viral attachment inhibitor and vaccine | Cellular \& Molecular Immunology.

21. Lan J, Ge J, Yu J, Shan S, Zhou H, et al. (2020) Structure of the SARS-CoV-2 spike receptor-binding domain bound to the ACE2 receptor. Nature.

22. Nagpal K, Singh SK, Mishra DN (2010) Chitosan Nanoparticles: A Promising System in Novel Drug Delivery. Chem Pharm Bull (Tokyo) 58: 1423.

23. Wang JJ, Zeng ZW, Xiao RZ, Xie T, Zhou GL, et al. (2011) Recent advances of chitosan nanoparticles as drug carriers. Int J Nanomedicine 6: 765.

24. Rytting E, Nguyen J, Wang X, Kissel T (2008) Biodegradable polymeric nanocarriers for pulmonary drug delivery, Expert Opin. Drug Deliv 5: 629.

ISSN: 2574-1241

DOI: 10.26717/BJSTR.2021.37.005960

Uysal Melike. Biomed J Sci \& Tech Res

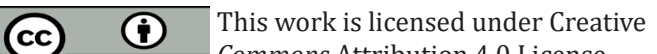

Submission Link: https://biomedres.us/submit-manuscript.php
25. Islam N, Ferro (2016) Recent advances in chitosan-based nanoparticulate pulmonary drug delivery, Nanoscale 8: 14341.

26. Chen MC, Mi FL, Liao ZX, Hsiao CW, Sonaje K, et al. (2013) Recent advances in chitosan-based nanoparticles for oral delivery of macromolecules. Adv Drug Deliv Rev 65: 865.

27. Mohammed MA, Syeda JTM, Wasan KM, Wasan EK (2017) An Overview of Chitosan Nanoparticles and Its Application in Non-Parenteral Drug Delivery, Pharmaceutics 9.

28. Schiller JL, Fogle MM, Bussey O, Kissner WJ, Hill DB, et al. (2020) Antibody-mediated trapping in biological hydrogels is governed by sugar-sugar hydrogen bonds. Acta Biomater 107: 91.

29. Braunstein M, Hickey AJ, Ekins S (2019) Why Wait? The Case for Treating Tuberculosis with Inhaled Drugs. Pharm Res 36: 166.

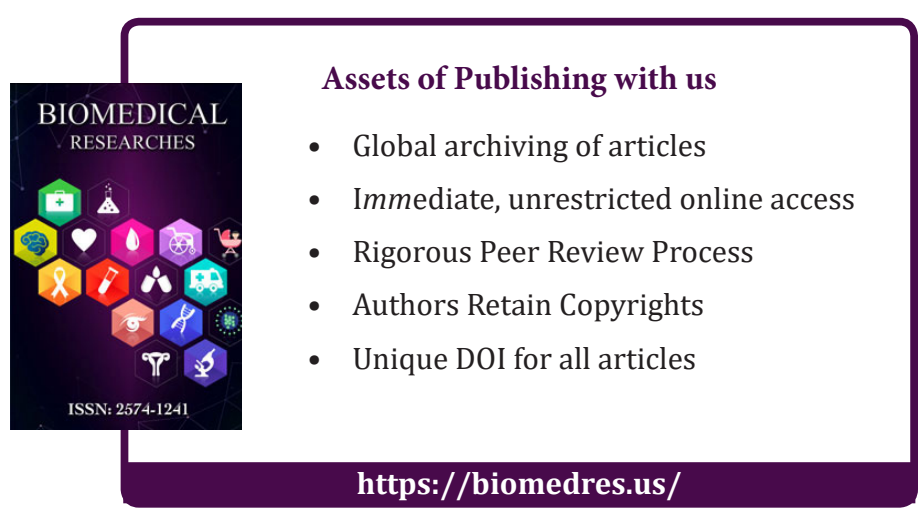

